\title{
Knowledge of Pharmacists about Scientific Publications in Saudi Arabia
}

\author{
Yousef Ahmed Alomi* iD, BSC. \\ Pharm, MSc. Clin Pharm, BCPS, BCNSP, \\ DiBA, CDE, Critical Care Clinical Pharmacists, \\ TPN Clinical Pharmacist, Freelancer \\ Business Planner, Content Editor and Data \\ Analyst, Riyadh, Saudi Arabia. \\ Sultan Mohammed Al-Jarallah, \\ Bsc. Pahrm, Msc Clin Pharm, Head, \\ Ambulatory Care Pharmacy, Oncology \\ and Hematology Clinical Pharmacist, \\ Pharmaceutical Care Department, Security \\ Forces Hospital, Riyadh, SAUDI ARABIA. \\ Juman saad Mohammad Alsaab, \\ Erada psychiatric Hospital-Alkharj, Riyadh, \\ SAUDI ARABIA.
}

Razan Alshehri, College of Pharmacy, Taif University, Tail, SAUDI ARABIA.

Khawla Ibrahim Al-shahrani, College of Pharmacy, Taif University, Tail, SAUDI ARABIA.

\section{Correspondence:}

Dr. Yousef Ahmed Alomi, BSc. Pharm, MSc. Clin Pharm, BCPS, BCNSP, DiBA, CDE, Critical Care Clinical Pharmacists, TPN Clinical Pharmacist, Freelancer Business Planner, Content Editor and Data Analyst, Riyadh 11392, Riyadh, Saudi Arabia.

Phone no: +966504417712

E-mail: yalomi@gmail.com
Received: 22-04-2021;

Accepted: 05-07-2021.

Copyright: (c) the author(s),publisher and licensee Pharmacology, Toxicology and Biomedical Reports. This is an open-access article distributed under the terms of the Creative Commons Attribution NonCommercial License, which permits unrestricted non-commercial use, distribution, and reproduction in any medium, provided the original work is properly cited.

This is an open access article distributed under the terms of the Creative Commons AttributionNonCommercial-ShareAlike 4.0 License

Access this article online

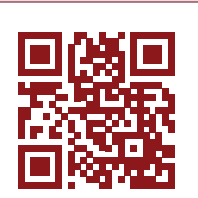

www.ptbreports.org

DOI:

10.5530/PTB.2021.7.19

\begin{abstract}
Objectives: In this study, we aimed to assess pharmacists' knowledge about scientific publications in the Kingdom of Saudi Arabia. Methods: In this cross-sectional survey, we analyzed pharmacists' knowledge about scientific publications in Saudi Arabia. We used a self-reported electronic survey questionnaire and distributed it to pharmacists from interns to consultants and specialists. The survey collected demographic information of the responders and their knowledge of selected research paper elements in a scientific journal. We used 5-point Likert response scale system with closeended questions to obtain responses. The data were collected through the Survey Monkey system and analyzed using the Statistical Package of Social Sciences (SPSS) and Jeffery's Amazing Statistics Program (JASP), and Microsoft Excel (version 16) software. Results: A total of 543 pharmacists responded to the questionnaire. Of them, more than one-quarter of the participants responded from the central region $(155(28.55 \%))$, followed by the eastern region $(133(24.49 \%))$, with statistically significant differences between all regions $(p=0.000)$. Females responded more $(321(59.12 \%))$ than that of males (222 $(40.88 \%))$. The majority of the responders were Saudi nationals (351 $(64.64 \%))$, followed by non-Saudi nationals (192 $(35.36 \%))$, with statistically significant differences between them $(p=0.000)$. The average score for knowledge of pharmacists about writing a section in the research article was 4.07, with high scores obtained for the elements "knowledge of the abstract section" (4.51) and "knowledge of the Introduction section" (4.47), with statistically significant between all responses $(p=0.000)$. The average score for knowledge of pharmacists about various study designs in the manuscript was 3.36, with high scores obtained for the elementary knowledge of cohort study (3.59), case series (3.59), observational study, and Letters to the editor (3.49), with statistically significant differences between responses $(p=0.000)$. The average score for knowledge of pharmacists about journal indexing database was (3.17), with high scores obtained for the elementary knowledge of the Google Scholar (3.78), PubMed (3.60), and Index Medicus (3.43). The scores for the reliability analysis of McDonald's $\omega$ was (0.843), Cronbach's $\alpha$ was (0.847), Gutmann's $\lambda 2$ was (0.888), Gutmann's was $\lambda 6$ (0.985), and Greater Lower Bound was (0.994). Conclusion: Pharmacists' knowledge about writing research sections, study design, and journal indexing database for scientific publications in Saudi Arabia was varied. Therefore, we highly recommend improving pharmacists' training and education during graduation to improve patients' pharmaceutical care in Saudi Arabia.

Key words: Knowledge, Pharmacist, Scientific Publications, Saudi Arabia.
\end{abstract}

\section{INTRODUCTION}

Pharmacists gain their pharmacy or medical knowledge from various resources, such as textbooks, journals, websites, and social media. Several types of research designs can be used to write an article, namely, narrative review, systematic review, and observational studies, in addition to clinical trials, cases series, and case reports. ${ }^{1}$ Each type of article in biomedical journals consists of the following sections: Introduction, Materials and Methods, Results, Discussion, Conclusion, and References. ${ }^{2}$ Different international publications guidelines are recommended to follow the entire structure. $^{2,3}$ Various guidelines for writing scientific research recommend all healthcare professionals, including pharmacists, follow the structure of writing scientific research. Those guidelines were designed based on research methodology. For instant, clinical trials, observational studies, epidemiology research, and pharmacoepidemiology. Consequently, the research paper authors should be educated and trained on writing different types of research articlesintended for publication. ${ }^{4-8}$ The pharmacist should be aware of that knowledge of writing research for publications. Most pharmacists are trained to write publications during the school of pharmacy period through academic assay or academic writing. ${ }^{9}$ The pharmacy school has changed its curriculum from a bachelor's degree to a pharm D degree in Saudi Arabia and other Middle East countries. ${ }^{9,10}$ Those changes included multiple things, including the research projects. Each study has to do a research project before graduation. Moreover, the pharmacy practice program required a research project for graduation. ${ }^{9}$ The Saudi Commission Health Specialties (SCHS) recommended making research or publications considered suggested scores for residency admissions. Besides, the healthcare organizations recommended doing research and publications during practice. ${ }^{11,12}$ In addition, some pharmacists get research and scientific publication volunteer courses.

Much of the researches have been done annually. ${ }^{13}$ However, few of them had been published in 
peer-reviewed biomedical journals. ${ }^{14}$ That is related to inadequate basic research knowledge or insufficient knowledge of biostatistics analysis that is required for scientific publications. ${ }^{15-18}$ A previous study has reported the essential Awareness elements of scientific research, ${ }^{19}$ and others have discussed ethics in research, biostatistics knowledge of healthcare professionals, and resources that can be used. ${ }^{20,21}$ However, to the best of our knowledge, no studies are conducted to discuss pharmacists' knowledge about scientific publications. ${ }^{22}$ Therefore, in this study, we aimed to declare the knowledge of pharmacists about scientific publications in the Kingdom of Saudi Arabia.

\section{METHODS}

This is a 6-month cross-sectional study conducted to assess pharmacists' knowledge about scientific publications in Saudi Arabia. We used a self-reported electronic survey questionnaire and distributed it to pharmacists from interns to consultants and specialists in Saudi Arabia. All non-pharmacists, students, and incomplete surveys were excluded from this study. The survey collected demographic information of the responders and their knowledge of writing selected elements of a manuscript for a scientific journal and their knowledge of study designs, journal indexing database, and reference management software tools. We used a 5-point Likert response scale system with close-ended questions to obtain responses. This cross-sectional study calculated the sample size according to the previous literature with unlimited population size. The confidence was on the level of 95\%, a $z$ score of 1.96 , a margin of error of $5 \%$, and the population percentage was $50 \%$. Besides, the drop-out rate was $10 \%$. Consequently, the sample size was calculated as 418 with a power of study of $80 \% \cdot{ }^{23-25}$ The response rate required for this sample size was at least $60-70 \% .{ }^{25,26}$ The survey was distributed through social media such as WhatsApp and Telegram and via face-to-face contact. In addition, a reminder message was sent once every 1-2 weeks. Expert reviewers and pilot testing validated the survey. Furthermore, various reliability tests such as McDonald's $\omega$, Cronbach's $\alpha$, Gutmann's $\lambda 2$, and Gutmann's $\lambda 6$ were conducted. The data were collected through the Survey Monkey system and analyzed using Microsoft Excel (version 16), Statistical Package of Social Sciences (SPSS), and Jeffery's Amazing Statistics Program (JASP). We performed descriptive and frequency analysis, the goodness of fit, correlation, and inferential analyses on factors affecting pharmacists' knowledge about scientific publications. The STROBE (Strengthening the reporting of observational studies in epidemiology statement: Guidelines for reporting observational studies) guided the reporting of the results of this study. ${ }^{2-4}$

\section{RESULTS}

A total number of 543 pharmacists responded to the questionnaire. Of them, more than one-quarter responded from the central region $(155(28.55 \%))$ and eastern region (133 (24.49\%)), with statistically significant differences between the provinces $(p=0.000)$. Most of the responders were from private primary healthcare centers $(110(20.26 \%))$ and Ministry of Health (MOH) hospitals (97 (17.86\%)), with a statistically significant difference between working sites $(p=0.000)$. Females responded more than males (321 (59.12\%) versus $222(40.88 \%))$. Based on nationality, Saudi nationals responded more than non-Saudi nationals (351 (64.64\%) versus $192(35.36 \%))$, with statistically significant differences between nationalities $(p=0.000)$. Most of the responders were in the age group of 30-44 years (209 (38.49\%)) and $18-29$ years $(166(30.57 \%))$, with statistically significant differences between all age groups $(p=0.000)$. Most of the pharmacists were community pharmacists $(107(19.74 \%))$ and pharmacy supervisors (57 $(10.52 \%))$, with statistically significant differences between all levels $(p=0.000)$. Most of the responders held Diploma in Pharmacy
(202 (37.20\%)), Bachelor in Pharmacy (199 (36.65\%)), and Master of Science in Clinical Pharmacy (140 (25.78\%)). Most pharmacists had a work experience of $6-10$ years $(140(34.15 \%))$ and $3-5$ years (124 (30.24\%)), with a statistically significant difference between years of experience $(p=0.000)$. More than two-thirds of the responders had a certification from one board of pharmaceutical specialties (367 (68.21\%)). Of them, 220 (40.89\%) were Board-Certified Critical Care specialists, 218 (40.52\%)were Board-Certified Nuclear Pharmacists, 192 (35.69\%) were Board-Certified Ambulatory Care specialists, and 187 (34.76\%) were Board-Certified Nutrition Support specialists. Most of the pharmacists practiced in the area of narcotics (61 (11.25\%)), clinical pharmacy (59 $(10.89 \%))$, and repacking $(57(10.52 \%))$, with statistically significant difference between all sites of pharmacy practice $(p=0.000)$. There was a medium positive correlation between age (years) and years of experience based on Kendall's tau_b (0.414) and Spearman's rho (0.485) correlation coefficients, with a statistically significant difference between the two factors $(p<0.001)$. There was a medium positive correlation between site of work and current position held based on Kendall's tau_b (0.457) and Spearman's rho (0.610), with a statistically significant difference between the two factors $(p<0.001)$ (Tables 1 and 2$)$.

The average score for pharmacists' knowledge in writing a section in the manuscript was 4.07, with high scores obtained for knowledge of the Abstract section (4.51) and knowledge of the Introduction section (4.47). In comparison, the lowest scores were obtained for Limitations (3.63) and References section (3.82), with statistically significant differences between responses. Moreover, all aspects showed statistically significant differences between responses $(p<0.001)$ (Table 3$)$. The total average scores of pharmacist knowledge in publishing various study designs in the manuscript were (3.36), with high scores obtained for the element knowledge of the cohort (3.59), case series (3.59), observational study, and letters to the editor (3.49). In comparison, the lowest scores were obtained for meta-analysis (3.11), quality improvement study (3.15), and pre-clinical animal study (3.17), with statistically significant differences between the responses. Moreover, all aspects were statistically significant $(p<0.001)$ (Table 4$)$. The average score for knowledge of pharmacists about journal indexing database was 3.17 with high scores obtained for the element knowledge of the Google Scholar (3.78), PubMed (3.60), and Index Medicus (3.43). In comparison, the lowest scores were obtained for the Scilit database (2.92), and Directory of Research Journals Indexing (2.95), and World Cat (OCLC) (2.96), with statistically significant between responses. Moreover, all aspects were statistically significant $(p<0.001)$ (Table 5). The total average score for pharmacist knowledge of using reference management software tools was 3.47, with high scores obtained for the element knowledge of the Mendeley (3.64) and Qiqqa (3.63). In comparison, the lowest scores were obtained for Papers (3.15) and Colwiz (3.26), with statistically significant differences between responses. Moreover, all aspects were statistically significant $(p<0.001)$ (Table 6). The scores for reliability analysis of McDonald's $\omega$ was ( 0.843$)$, Cronbach's a was (0.847), Gutmann's $\lambda 2$ was (0.888), Gutmann's $\lambda 6$ was (0.985), and Greater Lower Bound was (0.994).

\section{Factors affecting the knowledge of pharmacists about scientific publications}

Factors affecting the knowledge of pharmacists about writing a section of the research paper. Using independent samples Kruskal-Wallis test and the Bonferroni correction for multiple tests, we adjusted the significant values. The factors that might affect pharmacists' knowledge in writing a section of the research paper include location, worksite, gender, age, practice area, current position held, and years of work experience. Two factors (nationality and position ) did not affect the knowledge of storage with a non-statistically significant difference $(p>0.05)$. Five locations impacted the knowledge of pharmacists about writing a section of the 
Alomi YA et.al. Knowledge of Scientific Publications in Saudi Arabia

\begin{tabular}{|c|c|c|c|}
\hline Locations & $\begin{array}{c}\text { Response } \\
\text { Count }\end{array}$ & $\begin{array}{c}\text { Response } \\
\text { Percent }\end{array}$ & $\begin{array}{c}p \text {-value } \\
(\mathrm{X} 2)\end{array}$ \\
\hline Central area & 155 & $28.55 \%$ & \multirow{5}{*}{0.000} \\
\hline North area & 115 & $21.18 \%$ & \\
\hline South area & 52 & $9.58 \%$ & \\
\hline East area & 133 & $24.49 \%$ & \\
\hline West area & 88 & $16.21 \%$ & \\
\hline Answered question & 543 & & \\
\hline Skipped question & 0 & & \\
\hline Site of work & $\begin{array}{c}\text { Response } \\
\text { Count }\end{array}$ & $\begin{array}{c}\text { Response } \\
\text { Percent }\end{array}$ & $\begin{array}{c}p \text {-value } \\
(\mathrm{X} 2)\end{array}$ \\
\hline Ministry of Health & 85 & $15.65 \%$ & \multirow{11}{*}{0.000} \\
\hline General Medical Directorate in Region & 68 & $12.52 \%$ & \\
\hline MOH government Hospital & 97 & $17.86 \%$ & \\
\hline Non- MOH government Hospital & 48 & $8.84 \%$ & \\
\hline MOH-Primary Care Center & 31 & $5.71 \%$ & \\
\hline Private Hospital & 16 & $2.95 \%$ & \\
\hline Private Primary Care Center & 110 & $20.26 \%$ & \\
\hline Community pharmacy & 48 & $8.84 \%$ & \\
\hline University & 27 & $4.97 \%$ & \\
\hline Pharmaceutical company & 8 & $1.47 \%$ & \\
\hline Non employment & 5 & $0.92 \%$ & \\
\hline Answered question & 543 & & \\
\hline Skipped question & $\mathbf{0}$ & & \\
\hline Gender & $\begin{array}{c}\text { Response } \\
\text { Count }\end{array}$ & $\begin{array}{c}\text { Response } \\
\text { Percent }\end{array}$ & \\
\hline Male & 222 & $40.88 \%$ & \multirow{2}{*}{0.000} \\
\hline Female & 321 & $59.12 \%$ & \\
\hline Answered question & 543 & & \\
\hline Skipped question & $\mathbf{0}$ & & \\
\hline Nationality & $\begin{array}{c}\text { Response } \\
\text { Count }\end{array}$ & $\begin{array}{c}\text { Response } \\
\text { Percent }\end{array}$ & \\
\hline Saudi & 351 & $64.64 \%$ & \multirow{2}{*}{0.000} \\
\hline Non-Saudi & 192 & $35.36 \%$ & \\
\hline Answered question & 543 & & \\
\hline Skipped question & 0 & & \\
\hline Age & $\begin{array}{c}\text { Response } \\
\text { Count }\end{array}$ & $\begin{array}{c}\text { Response } \\
\text { Percent }\end{array}$ & \\
\hline $18-29$ & 166 & $30.57 \%$ & \multirow{4}{*}{0.000} \\
\hline $30-44$ & 209 & $38.49 \%$ & \\
\hline $45-60$ & 137 & $25.23 \%$ & \\
\hline$>60$ & 31 & $5.71 \%$ & \\
\hline Answered question & 543 & & \\
\hline Skipped question & $\mathbf{0}$ & & \\
\hline
\end{tabular}

\begin{tabular}{|c|c|c|c|}
\hline Pharmacist's Qualifications & $\begin{array}{l}\text { Response } \\
\text { Count }\end{array}$ & $\begin{array}{c}\text { Response } \\
\text { Percent }\end{array}$ & $\begin{array}{l}p \text {-value } \\
(\mathrm{X} 2)\end{array}$ \\
\hline Diploma pharmacy & 46 & $8.47 \%$ & \\
\hline BSc. Pharm & 199 & $36.65 \%$ & \\
\hline M.S & 91 & $16.76 \%$ & \\
\hline MSc. Clinical Pharmacy & 140 & $25.78 \%$ & \\
\hline Pharm.D & 202 & $37.20 \%$ & \\
\hline $\mathrm{Ph} . \mathrm{D}$ & 98 & $18.05 \%$ & \\
\hline MBA & 83 & $15.29 \%$ & \\
\hline Pharmacy Residency Two years (R1) & 90 & $16.57 \%$ & \\
\hline Pharmacy Residency one year (R2) & 93 & $17.13 \%$ & \\
\hline Fellowship & 127 & $23.39 \%$ & \\
\hline Student pharmacist & 69 & $12.71 \%$ & \\
\hline Intern pharmacist & 23 & $4.24 \%$ & \\
\hline Answered question & 543 & & \\
\hline Skipped question & $\mathbf{0}$ & & \\
\hline $\begin{array}{l}\text { Board of Pharmacy Specialties } \\
\text { certificate }\end{array}$ & $\begin{array}{l}\text { Response } \\
\text { Count }\end{array}$ & $\begin{array}{c}\text { Response } \\
\text { Percent }\end{array}$ & \\
\hline $\begin{array}{l}\text { Board Certified Ambulatory Care } \\
\text { Pharmacist (BCACP) }\end{array}$ & 192 & $35.69 \%$ & \\
\hline $\begin{array}{l}\text { Board Certified Critical Care } \\
\text { Pharmacist (BCCCP) }\end{array}$ & 220 & $40.89 \%$ & \\
\hline $\begin{array}{l}\text { Board Certified Nuclear Pharmacist } \\
\text { (BCNP) }\end{array}$ & 218 & $40.52 \%$ & \\
\hline $\begin{array}{l}\text { Board Certified Nutrition Support } \\
\text { Pharmacist (BCNSP) }\end{array}$ & 187 & $34.76 \%$ & \\
\hline $\begin{array}{l}\text { Board-certified Oncology Pharmacist } \\
\text { (BCOP) }\end{array}$ & 39 & $7.25 \%$ & \\
\hline $\begin{array}{l}\text { Board Certified Pediatric Pharmacy } \\
\text { Specialist (BCPPS) }\end{array}$ & 58 & $10.78 \%$ & \\
\hline $\begin{array}{l}\text { Board Certified Pharmacotherapy } \\
\text { Specialists (BCPS) }\end{array}$ & 71 & $13.20 \%$ & \\
\hline $\begin{array}{l}\text { Board-certified Psychiatric } \\
\text { Pharmacist (BCPP) }\end{array}$ & 64 & $11.90 \%$ & \\
\hline Non & 171 & $31.78 \%$ & \\
\hline Answered question & 538 & & \\
\hline Skipped question & 5 & & \\
\hline Position Held & $\begin{array}{l}\text { Response } \\
\text { Count }\end{array}$ & $\begin{array}{c}\text { Response } \\
\text { Percent }\end{array}$ & \\
\hline $\begin{array}{l}\text { General Manager of Pharmaceutical } \\
\text { care }\end{array}$ & 13 & $2.40 \%$ & \multirow{4}{*}{0.000} \\
\hline $\begin{array}{l}\text { Manager of Pharmaceutical care at } \\
\text { the region }\end{array}$ & 49 & $9.04 \%$ & \\
\hline Director of Hospital pharmacy & 42 & $7.75 \%$ & \\
\hline Supervisor of pharmacy units & 57 & $10.52 \%$ & \\
\hline $\begin{array}{l}\text { Director of Primary care center } \\
\text { pharmacy }\end{array}$ & 38 & $7.01 \%$ & \\
\hline Pharmacy Technicians & 51 & $9.41 \%$ & \\
\hline Lecturer & 24 & $4.43 \%$ & \\
\hline Staff Pharmacist & 49 & $9.04 \%$ & \\
\hline
\end{tabular}

Continued... 


\begin{tabular}{|c|c|c|c|}
\hline Community Pharmacist & 107 & $19.74 \%$ & \\
\hline Clinical Pharmacist & 27 & $4.98 \%$ & \\
\hline Deputy Director of Pharmacy & 49 & $9.04 \%$ & \\
\hline Manager & 26 & $4.80 \%$ & \\
\hline $\begin{array}{l}\text { Pharmaceutical company } \\
\text { representative }\end{array}$ & 4 & $0.74 \%$ & \\
\hline Pharmaceutical company supervisor & 1 & $0.18 \%$ & \\
\hline Non employment & 5 & $0.92 \%$ & \\
\hline Answered question & 542 & & \\
\hline Skipped question & 1 & & \\
\hline $\begin{array}{l}\text { Years of experience at Dentists } \\
\text { career }\end{array}$ & $\begin{array}{l}\text { Response } \\
\text { Count }\end{array}$ & $\begin{array}{c}\text { Response } \\
\text { Percent }\end{array}$ & \\
\hline$<3$ & 64 & $15.61 \%$ & \multirow{5}{*}{0.000} \\
\hline $3-5$ & 124 & $30.24 \%$ & \\
\hline $6-10$ & 140 & $34.15 \%$ & \\
\hline $11-15$ & 65 & $15.85 \%$ & \\
\hline$>15$ & 17 & $4.15 \%$ & \\
\hline Answered question & 410 & & \\
\hline Skipped question & 133 & & \\
\hline Pharmacy practice area & $\begin{array}{l}\text { Response } \\
\text { Count }\end{array}$ & $\begin{array}{c}\text { Response } \\
\text { Percent }\end{array}$ & \\
\hline Inpatient Pharmacy & 51 & $9.41 \%$ & \multirow{18}{*}{0.000} \\
\hline Outpatient Pharmacy & 38 & $7.01 \%$ & \\
\hline Satellite Pharmacy & 45 & $8.30 \%$ & \\
\hline Narcotics & 61 & $11.25 \%$ & \\
\hline Extemporaneous Preparation & 28 & $5.17 \%$ & \\
\hline Clinical Pharmacy & 59 & $10.89 \%$ & \\
\hline Inventory Control & 34 & $6.27 \%$ & \\
\hline Drug Information & 4 & $0.74 \%$ & \\
\hline Emergency pharmacy & 39 & $7.20 \%$ & \\
\hline Medication safety & 39 & $7.20 \%$ & \\
\hline Repacking & 57 & $10.52 \%$ & \\
\hline Pharmacy Education and Training & 24 & $4.43 \%$ & \\
\hline Pharmacy Research & 15 & $2.77 \%$ & \\
\hline Primary care pharmacy & 28 & $5.17 \%$ & \\
\hline Community pharmacy & 9 & $1.66 \%$ & \\
\hline Pharmaceutical company & 6 & $1.11 \%$ & \\
\hline Regulation/Administration & 1 & $0.18 \%$ & \\
\hline Non employment & 4 & $0.74 \%$ & \\
\hline Answered question & 543 & & \\
\hline Skipped question & $\mathbf{0}$ & & \\
\hline
\end{tabular}

research paper. The northern region obtained the lowest score (3.1207) with a statistically significant difference between all regions $(p=0.000)$. Females got a higher score than males (3.8244 versus3.7154), with a statistically significant difference between them $(p=0.032)$. In terms of age, the lowest score was (2.1875) obtained for the responders in the age group of 65-74 years, with a statistically significant difference between all age groups $(p=0.000)$. In terms of the worksite, low scores were obtained for those working at $\mathrm{MOH}$ (2.6029) and university (2.8565), with a statistically significant difference between all worksites $(p=0.000)$. In terms of practice areas, the lowest score (2.3824) was obtained for inpatient pharmacy, with a statistically significant difference between all worksites $(p=0.000)$. In terms of work experience, the lowest score (2.5588) was obtained for $>15$ years of experience, with a statistically significant difference between all levels $(p=0.000)$. In terms of positions held, the lowest score (2.4361) was obtained for director of a primary healthcare center and general manager of pharmaceutical care (2.4808), with a statistically significant difference between them $(p=0.000)$. A multiple regression analysis revealed the relationship between knowledge in writing sections of the research paper and factors affecting it. We measured knowledge as the dependent variable and factors as the expletory variable. The results showed a weak relationship $(\mathrm{R}=0.262$ with $p=0.000)$ between the two variables. Three out of seven factors showed non-significant differences $(p>0.05)$. However, a single factor (current position) explained a $19.6 \%$ positive relationship to the variation with a statistically significant difference $(p=0.002)$, which the Bootstrap model confirmed. Furthermore, the relationship was verified by the non-existence of multi-collinearity with the current position factor with Variance Inflation Factor (VIF) of 1.681, which is less than 3 or $5^{27-29}$ (Table 6).

Next, the factors affecting the knowledge of pharmacists about various study designs were tested. We adjusted the significant values by using independent samples Kruskal-Wallis test and the Bonferroni correction for multiple tests. Among the factors studied, a single factor (nationality) did not affect the knowledge of study designs, with a non-statistically significant difference $(p>0.05)$. Five locations affected to influence the knowledge of study design. The southern region obtained the lowest score (2.0247), followed by the northern region (2.2903), with a statistically significant difference between all regions $(p=0.000)$. Females were affected more than males (2.7316 versus 2.4054 ), with a statistically significant difference between them $(p=0.022)$. In terms of age, the lowest score was for the age group of 65-74 years (2.1012), with a statistically significant difference between all age groups $(p=0.000)$. In terms of the worksite, the lowest score was obtained for $\mathrm{MOH}$ (1.9896), with a statistically significant difference between all worksites $(p=0.000)$. In terms of practice area, the lowest score was obtained for inpatient pharmacy (1.8617), followed by pharmacy education (2.1149) and medications safety (2.1465), with a statistically significant difference between all areas $(p=0.000)$. In terms of work experience, the lowest score (1.6681) was obtained for $>15$ years of experience, with a statistically significant difference between them $(p=0.000)$. In terms of the position held, the lowest score was obtained for general manager of pharmaceutical care (1.4451), followed by director of primary care pharmacy (2.1071) and supervisor of pharmacy unit (2.0906), with a statistically significant difference between them $(p=0.000)$. The relationship between knowledge in publishing various study designs in a research paper and factors affecting it was studied. The multiple regression model revealed a medium relationship $(\mathrm{R}=0.458$ with $p=0.000)$ between knowledge in publishing various study designs in research papers and its factors. Three out of eight factors showed non-significant differences ( $p>0.05$ ). However, location explained $30.0 \%$ of the positive relationship, age explained $12.4 \%$ of the negative relationship, nationality explained $22.3 \%$ of the positive relationship, practice area explained $13.7 \%$ of the negative relationship, and current position held explained $26.7 \%$ of the positive relationship to the variation, with a statistically significant ( $p=0.000,0.011,0.000,0.009$ ), and 0.000 , respectively) the difference, which the Bootstrap model confirmed. The relationship was verified by the non-existence of multi-collinearity with the current position factor with VIF of $1.134,1.186,1.536), 1.381$, and 1.681, respectively), which is less than 3 or $5^{27-29}$ (Table 7 ). 


\begin{tabular}{|c|c|c|c|c|c|c|c|c|c|c|c|c|}
\hline \multicolumn{2}{|c|}{$\begin{array}{l}\text { Complete } \\
\text { information }\end{array}$} & \multicolumn{2}{|c|}{$\begin{array}{l}\text { Incomplete } \\
\text { information }\end{array}$} & \multicolumn{2}{|c|}{$\begin{array}{c}\text { Weak } \\
\text { information }\end{array}$} & \multicolumn{2}{|c|}{$\begin{array}{l}\text { I do not have } \\
\text { information }\end{array}$} & \multicolumn{2}{|c|}{$\begin{array}{l}\text { I do not need this } \\
\text { information }\end{array}$} & \multirow{2}{*}{$\begin{array}{l}\text { Total } \\
543 \\
\end{array}$} & \multirow{2}{*}{$\begin{array}{c}\text { Weighted } \\
\text { Average }\end{array}$} & \multirow{2}{*}{$\begin{array}{c}p \text {-value } \\
0.000 \\
\end{array}$} \\
\hline 355 & $65.38 \%$ & 133 & $24.49 \%$ & 36 & $6.63 \%$ & 17 & $3.13 \%$ & 2 & $0.37 \%$ & & & \\
\hline 303 & $55.80 \%$ & 203 & $37.38 \%$ & 27 & $4.97 \%$ & 8 & $1.47 \%$ & 2 & $0.37 \%$ & 543 & 4.47 & 0.000 \\
\hline 216 & $39.85 \%$ & 191 & $35.24 \%$ & 126 & $23.25 \%$ & 6 & $1.11 \%$ & 3 & $0.55 \%$ & 542 & 4.13 & 0.000 \\
\hline 205 & $37.75 \%$ & 236 & $43.46 \%$ & 85 & $15.65 \%$ & 16 & $2.95 \%$ & 1 & $0.18 \%$ & 543 & 4.16 & 0.000 \\
\hline 171 & $31.49 \%$ & 223 & $41.07 \%$ & 90 & $16.57 \%$ & 59 & $10.87 \%$ & 0 & $0.00 \%$ & 543 & 3.93 & 0.000 \\
\hline 144 & $26.52 \%$ & 194 & $35.73 \%$ & 100 & $18.42 \%$ & 69 & $12.71 \%$ & 36 & $6.63 \%$ & 543 & 3.63 & 0.000 \\
\hline 196 & $36.10 \%$ & 183 & $33.70 \%$ & 43 & $7.92 \%$ & 113 & $20.81 \%$ & 8 & $1.47 \%$ & 543 & 3.82 & 0.000 \\
\hline & & & & & & & & & & 543 & & \\
\hline & & & & & & & & & & 0 & & \\
\hline
\end{tabular}

Table 4: Level of knowledge in publishing various study designs.

\begin{tabular}{|c|c|c|c|c|c|c|c|c|c|c|c|c|c|}
\hline \multirow[b]{2}{*}{ Meta-analysis } & \multicolumn{2}{|c|}{$\begin{array}{c}\text { Complete } \\
\text { information }\end{array}$} & \multicolumn{2}{|c|}{$\begin{array}{l}\text { Incomplete } \\
\text { information }\end{array}$} & \multicolumn{2}{|c|}{$\begin{array}{c}\text { Weak } \\
\text { information }\end{array}$} & \multicolumn{2}{|c|}{$\begin{array}{l}\text { I do not have } \\
\text { information }\end{array}$} & \multicolumn{2}{|c|}{$\begin{array}{l}\text { I do not need } \\
\text { this information }\end{array}$} & \multirow{2}{*}{$\begin{array}{c}\text { Total } \\
543\end{array}$} & \multirow{2}{*}{\begin{tabular}{|c|}
$\begin{array}{c}\text { Weighted } \\
\text { Average }\end{array}$ \\
3.11 \\
\end{tabular}} & \multirow{2}{*}{$\begin{array}{r}p \text {-value } \\
0.000\end{array}$} \\
\hline & 78 & $14.36 \%$ & 202 & $37.20 \%$ & 103 & $18.97 \%$ & 24 & $4.42 \%$ & 136 & $25.05 \%$ & & & \\
\hline Systematic Review & 51 & $9.41 \%$ & 236 & $43.54 \%$ & 87 & $16.05 \%$ & 134 & $24.72 \%$ & 34 & $6.27 \%$ & 542 & 3.25 & 0.000 \\
\hline Cohort & 126 & $23.60 \%$ & 172 & $32.21 \%$ & 163 & $30.52 \%$ & 39 & $7.30 \%$ & 34 & $6.37 \%$ & 534 & 3.59 & 0.000 \\
\hline Case series & 133 & $24.49 \%$ & 168 & $30.94 \%$ & 163 & $30.02 \%$ & 44 & $8.10 \%$ & 35 & $6.45 \%$ & 543 & 3.59 & 0.000 \\
\hline Case control & 93 & $17.16 \%$ & 205 & $37.82 \%$ & 156 & $28.78 \%$ & 26 & $4.80 \%$ & 62 & $11.44 \%$ & 542 & 3.44 & 0.000 \\
\hline Observational study & 97 & $17.86 \%$ & 188 & $34.62 \%$ & 175 & $32.23 \%$ & 49 & $9.02 \%$ & 34 & $6.26 \%$ & 543 & 3.49 & 0.000 \\
\hline Randomized controlled trail & 113 & $20.81 \%$ & 174 & $32.04 \%$ & 163 & $30.02 \%$ & 48 & $8.84 \%$ & 45 & $8.29 \%$ & 543 & 3.48 & 0.000 \\
\hline Letter to the editor & 110 & $20.30 \%$ & 189 & $34.87 \%$ & 153 & $28.23 \%$ & 37 & $6.83 \%$ & 53 & $9.78 \%$ & 542 & 3.49 & 0.000 \\
\hline General review & 73 & $13.70 \%$ & 198 & $37.15 \%$ & 150 & $28.14 \%$ & 68 & $12.76 \%$ & 44 & $8.26 \%$ & 533 & 3.35 & 0.000 \\
\hline Clinical practice guidelines & 62 & $11.70 \%$ & 162 & $30.57 \%$ & 214 & $40.38 \%$ & 49 & $9.25 \%$ & 43 & $8.11 \%$ & 530 & 3.28 & 0.000 \\
\hline Skipped & & & & & & & & & & & 0 & & \\
\hline
\end{tabular}

\begin{tabular}{|c|c|c|c|c|c|c|c|c|c|c|c|c|c|}
\hline Google scholar & \multicolumn{2}{|c|}{$\begin{array}{c}\text { Complete } \\
\text { information }\end{array}$} & \multicolumn{2}{|c|}{$\begin{array}{l}\text { Incomplete } \\
\text { information }\end{array}$} & \multicolumn{2}{|c|}{$\begin{array}{c}\text { Weak } \\
\text { information }\end{array}$} & \multicolumn{2}{|c|}{$\begin{array}{l}\text { I do not have } \\
\text { information }\end{array}$} & \multicolumn{2}{|c|}{$\begin{array}{l}\text { I do not need } \\
\text { this information }\end{array}$} & $\begin{array}{c}\text { Total } \\
543\end{array}$ & $\begin{array}{c}\text { Weighted } \\
\text { Average }\end{array}$ & $\frac{p \text {-value }}{0.000}$ \\
\hline Index medicus & 70 & $12.92 \%$ & 246 & $45.39 \%$ & 105 & $19.37 \%$ & 90 & $16.61 \%$ & 31 & $5.72 \%$ & 542 & 3.43 & 0.000 \\
\hline PubMed & 113 & $20.85 \%$ & 173 & $31.92 \%$ & 182 & $33.58 \%$ & 73 & $13.47 \%$ & 1 & $0.18 \%$ & 542 & 3.60 & 0.000 \\
\hline Web of sciences & 90 & $16.61 \%$ & 132 & $24.35 \%$ & 126 & $23.25 \%$ & 167 & $30.81 \%$ & 27 & $4.98 \%$ & 542 & 3.17 & 0.000 \\
\hline Chemical Abstracts & 82 & $15.10 \%$ & 113 & $20.81 \%$ & 115 & $21.18 \%$ & 218 & $40.15 \%$ & 15 & $2.76 \%$ & 543 & 3.05 & 0.000 \\
\hline $\begin{array}{l}\text { Directory of Research Journals } \\
\text { Indexing }\end{array}$ & 61 & $11.23 \%$ & 100 & $18.42 \%$ & 154 & $28.36 \%$ & 208 & $38.31 \%$ & 20 & $3.68 \%$ & 543 & 2.95 & 0.000 \\
\hline Microsoft Academics & 83 & $15.29 \%$ & 103 & $18.97 \%$ & 141 & $25.97 \%$ & 200 & $36.83 \%$ & 16 & $2.95 \%$ & 543 & 3.07 & 0.000 \\
\hline Answered & & & & & & & & & & & 543 & & \\
\hline Skipped & & & & & & & & & & & $\mathbf{0}$ & & \\
\hline
\end{tabular}


Alomi YA et.al. Knowledge of Scientific Publications in Saudi Arabia

\begin{tabular}{|c|c|c|c|c|c|c|c|c|c|c|c|c|c|}
\hline \multirow[b]{2}{*}{ Mendeley } & \multicolumn{2}{|c|}{$\begin{array}{l}\text { Complete } \\
\text { information }\end{array}$} & \multicolumn{2}{|c|}{$\begin{array}{l}\text { Incomplete } \\
\text { information }\end{array}$} & \multicolumn{2}{|c|}{ Weak information } & \multicolumn{2}{|c|}{$\begin{array}{l}\text { I do not have } \\
\text { information }\end{array}$} & \multicolumn{2}{|c|}{$\begin{array}{l}\text { I do not need this } \\
\text { information }\end{array}$} & \multirow{2}{*}{$\begin{array}{l}\text { Total } \\
542\end{array}$} & \multirow{2}{*}{$\begin{array}{c}\text { Weighted } \\
\text { Average }\end{array}$} & \multirow{2}{*}{$\frac{p \text {-value }}{0.000}$} \\
\hline & 170 & $31.37 \%$ & 144 & $26.57 \%$ & 124 & $22.88 \%$ & 70 & $12.92 \%$ & 34 & $6.27 \%$ & & & \\
\hline EndNote & 79 & $14.55 \%$ & 231 & $42.54 \%$ & 147 & $27.07 \%$ & 76 & $14.00 \%$ & 10 & $1.84 \%$ & 543 & 3.54 & 0.000 \\
\hline RefWork & 125 & $23.36 \%$ & 193 & $36.07 \%$ & 116 & $21.68 \%$ & 93 & $17.38 \%$ & 8 & $1.50 \%$ & 535 & 3.62 & 0.000 \\
\hline Zotero & 173 & $31.92 \%$ & 127 & $23.43 \%$ & 129 & $23.80 \%$ & 84 & $15.50 \%$ & 29 & $5.35 \%$ & 542 & 3.61 & 0.000 \\
\hline Qiqqa & 171 & $31.67 \%$ & 128 & $23.70 \%$ & 120 & $22.22 \%$ & 113 & $20.93 \%$ & 8 & $1.48 \%$ & 540 & 3.63 & 0.000 \\
\hline JabRef & 85 & $15.65 \%$ & 152 & $27.99 \%$ & 202 & $37.20 \%$ & 92 & $16.94 \%$ & 12 & $2.21 \%$ & 543 & 3.38 & 0.000 \\
\hline Colwiz & 68 & $12.62 \%$ & 192 & $35.62 \%$ & 118 & $21.89 \%$ & 134 & $24.86 \%$ & 27 & $5.01 \%$ & 539 & 3.26 & 0.000 \\
\hline Papers & 94 & $17.31 \%$ & 57 & $10.50 \%$ & 245 & $45.12 \%$ & 130 & $23.94 \%$ & 17 & $3.13 \%$ & 543 & 3.15 & 0.000 \\
\hline Answered & & & & & & & & & & & 543 & & \\
\hline Skipped & & & & & & & & & & & 0 & & \\
\hline
\end{tabular}

\begin{tabular}{|c|c|c|c|c|c|c|c|c|c|c|c|c|c|c|}
\hline \multicolumn{2}{|r|}{ Model } & R & $\begin{array}{c}\mathrm{R} \\
\text { Square }\end{array}$ & $F$ & Sig. & \multicolumn{2}{|c|}{$\begin{array}{l}\text { Unstandardized } \\
\text { Coefficients }\end{array}$} & $\begin{array}{c}\begin{array}{c}\text { Standardized } \\
\text { Coefficients }\end{array} \\
\text { Beta }\end{array}$ & $t$ & Sig. & \multicolumn{2}{|c|}{$\begin{array}{l}95.0 \% \text { Confidence } \\
\text { Interval for B }\end{array}$} & \multicolumn{2}{|c|}{$\begin{array}{l}\text { Collinearity } \\
\text { Statistics }\end{array}$} \\
\hline \multirow[t]{7}{*}{1} & (Constant) & \multirow[t]{7}{*}{$.262^{\mathrm{b}}$} & \multirow[t]{7}{*}{.069} & \multirow[t]{7}{*}{3.683} & \multirow[t]{7}{*}{$.000^{\mathrm{b}}$} & 2.914 & 0.377 & & 7.723 & 0.000 & 2.172 & 3.656 & & \\
\hline & Locations & & & & & 0.154 & 0.048 & 0.163 & 3.174 & 0.002 & 0.059 & 0.249 & 0.882 & 1.134 \\
\hline & Age (years) & & & & & -0.016 & 0.033 & -0.026 & -0.496 & 0.620 & -0.081 & 0.048 & 0.844 & 1.186 \\
\hline & Nationality & & & & & -0.036 & 0.173 & -0.012 & -0.208 & 0.836 & -0.375 & 0.304 & 0.651 & 1.536 \\
\hline & Sex & & & & & 0.075 & 0.154 & 0.026 & 0.487 & 0.626 & -0.228 & 0.378 & 0.818 & 1.222 \\
\hline & Practice area & & & & & -0.012 & 0.018 & -0.040 & -0.705 & 0.481 & -0.047 & 0.022 & 0.724 & 1.381 \\
\hline & Current Position & & & & & 0.076 & 0.024 & 0.196 & 3.134 & 0.002 & 0.028 & 0.124 & 0.595 & 1.681 \\
\hline
\end{tabular}

a. Dependent Variable: Pharmacist,s knowledge in writing sections of research paper, Predictors ${ }^{\text {b}}$ : (Constant), Location, Site of work, Age (years), Nationality, Pharmacist gender, Practice area, Current Position, and pharmacist experiances.

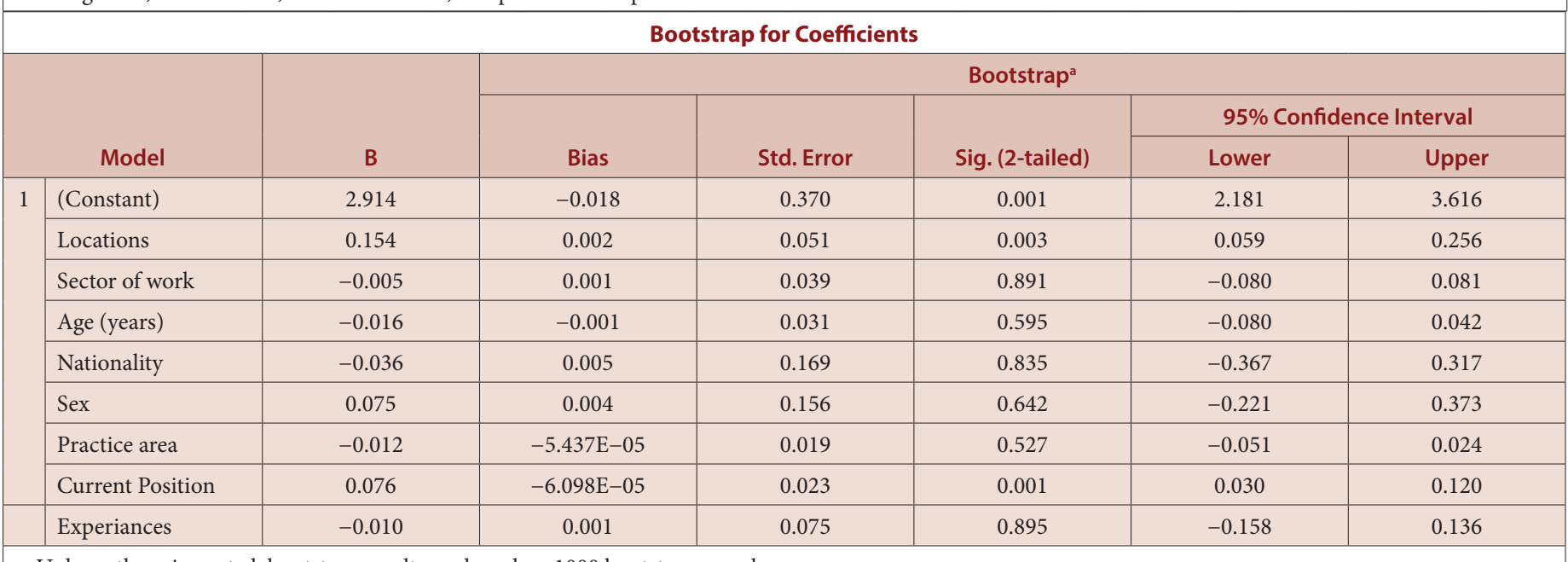

a. Unless otherwise noted, bootstrap results are based on 1000 bootstrap samples

Next, factors affecting the knowledge of the journal indexing database were analyzed using independent samples Kruskal-Wallis test and the Bonferroni correction for multiple tests. Various factors influenced the knowledge of the journal indexing database, including location, worksite, gender, age, practice area, current position held, and years of work experience. Five locations affected the knowledge of the journal indexing database. The western region obtained the lowest score (2.5601), followed by the southern region (2.6294), with a statistically significant difference between them $(p=0.000)$. In terms of nationality, Saudi nationals obtained a score of 4.9743 , with a statistically significant 
difference between them $(p=0.000)$. Females obtained a higher score than males (2.9476 versus 2.6491), with a statistically significant difference between them $(p=0.000)$. In terms of age, the lowest score (2.1319) was obtained for the age group of 65-74 years, with a statistically significant difference between them $(p=0.000)$. In terms of the worksite, the lowest score (2.2359) was obtained for the general medical directorate and non$\mathrm{MOH}$ governmental hospitals (2.2235), with a statistically significant difference between them $(p=0.000)$. In terms of practice area, the lowest score (2.2481) was obtained for satellite pharmacy followed by primary healthcare pharmacy (2.2887), with a statistically significant difference $(p=0.000)$. In terms of work experience, the lowest score (2.3346) was obtained for 11-15 years of experience, with a statistically significant difference between all levels $(p=0.000)$. In terms of the position held, the lowest score (2.1412) was obtained for the manager, clinical pharmacist (2.1341), and the director of hospital pharmacy (2.3075), with a statistically significant difference between all positions $(p=0.000)$. A multiple regression model revealed the relationship between the knowledge about journal indexing databases and factors affecting it. According to the results, there was a weak relationship $(\mathrm{R}=0.360$ with $p=0.000$ ) between knowledge about journal indexing databases and factors affecting them. Three out of eight factors showed nonsignificant differences ( $p>0.05$ ). However, location explained $15.9 \%$ of the negative relationship, nationality explained $26.5 \%$ of the positive relationship, gender explained $14.8 \%$ of the positive relationship, current position held explained $18 \%$ of the positive relationship, and years of experience explained $14.2 \%$ of the negative relationship to the variation, with a statistically significant $(p=0.001,0.000,0.004,0.003$, and 0.011 , respectively) difference, which was confirmed by Bootstrap model. The non-existence of multi-collinearity verified the relationship with the current position factor with VIF of 1.134, 1.536, 1.222, 1.681, and 1.397, respectively), which is less than 3 or $5^{21-23}$ (Table 8).

Next, the factors affecting the knowledge of pharmacists about reference management software were studied using independent samples KruskalWallis test and the Bonferroni correction for multiple tests. Various factors affected pharmacists' knowledge, such as location, worksite, gender, age, practice area, current position held, and years of experience. Based on the results, 5 locations affected the knowledge of pharmacists about the reference management software. The central region obtained a score of (5.0357), and the southern region got (5.2933) with a statistically significant difference between them $(p=0.007)$. In terms of nationality, Saudi nationals obtained the highest score (2.7320), with a statistically significant difference $(p=0.000)$. In terms of age, pharmacists in the age group of $>75$ years had the greatest knowledge of reference management software (3.5000), with a statistically significant difference $(p=0.000)$. In terms of the worksite, the lowest score was obtained for private primary healthcare centers (3.9148), with a statistically significant difference

\section{Table 8: Multiple regression of Factors with the knowledge in publishing various study designs in research paper. ${ }^{\mathrm{a}}$}

\begin{tabular}{|c|c|c|c|c|c|c|c|c|c|c|c|c|c|c|}
\hline \multirow{2}{*}{\multicolumn{2}{|c|}{ Model }} & \multirow[b]{2}{*}{$\mathbf{R}$} & \multirow[b]{2}{*}{$\begin{array}{c}\mathbf{R} \\
\text { Square }\end{array}$} & \multirow[b]{2}{*}{$F$} & \multirow[b]{2}{*}{ Sig. } & \multicolumn{2}{|c|}{$\begin{array}{l}\text { Unstandardized } \\
\text { Coefficients }\end{array}$} & \multirow{2}{*}{$\begin{array}{c}\begin{array}{c}\text { Standardized } \\
\text { Coefficients }\end{array} \\
\text { Beta }\end{array}$} & \multirow[b]{2}{*}{$t$} & \multirow[b]{2}{*}{ Sig. } & \multicolumn{2}{|c|}{$\begin{array}{l}95.0 \% \text { Confidence } \\
\text { Interval for B }\end{array}$} & \multicolumn{2}{|c|}{$\begin{array}{l}\text { Collinearity } \\
\text { Statistics }\end{array}$} \\
\hline & & & & & & B & $\begin{array}{l}\text { Std. } \\
\text { Error }\end{array}$ & & & & $\begin{array}{l}\text { Lower } \\
\text { Bound }\end{array}$ & $\begin{array}{l}\text { Upper } \\
\text { Bound }\end{array}$ & Tolerance & VIF \\
\hline \multirow[t]{7}{*}{1} & (Constant) & \multirow[t]{7}{*}{$.458^{\mathrm{b}}$} & \multirow[t]{7}{*}{.210} & \multirow[t]{7}{*}{13.362} & \multirow[t]{7}{*}{$.000^{\mathrm{b}}$} & 1.327 & 0.234 & & 5.667 & 0.000 & 0.867 & 1.787 & & \\
\hline & Locations & & & & & 0.191 & 0.030 & 0.300 & 6.345 & 0.000 & 0.132 & 0.250 & 0.882 & 1.134 \\
\hline & Sector of work & & & & & -0.036 & 0.021 & -0.105 & -1.657 & 0.098 & -0.078 & 0.007 & 0.489 & 2.043 \\
\hline & Nationality & & & & & 0.434 & 0.107 & 0.223 & 4.051 & 0.000 & 0.223 & 0.645 & 0.651 & 1.536 \\
\hline & Sex & & & & & 0.164 & 0.096 & 0.084 & 1.709 & 0.088 & -0.025 & 0.352 & 0.818 & 1.222 \\
\hline & Practice area & & & & & -0.029 & 0.011 & -0.137 & -2.622 & 0.009 & -0.050 & -0.007 & 0.724 & 1.381 \\
\hline & Current Position & & & & & 0.070 & 0.015 & 0.267 & 4.632 & 0.000 & 0.040 & 0.099 & 0.595 & 1.681 \\
\hline
\end{tabular}

a. Dependent Variable: Pharmacist's knowledge in publishing various study designs in research paper, Predictors ${ }^{\text {b: }}$ (Constant), Location, Site of work, Age (years), Nationality, Pharmacist gender, Practice area, Current Position, and pharmacist experiences.

\begin{tabular}{|c|c|c|c|c|c|c|c|}
\hline \multicolumn{8}{|c|}{ Bootstrap for Coefficients } \\
\hline \multirow{3}{*}{\multicolumn{2}{|c|}{ Model }} & \multirow[b]{3}{*}{ B } & \multicolumn{5}{|c|}{ Bootstrap ${ }^{a}$} \\
\hline & & & \multirow[b]{2}{*}{ Bias } & \multirow[b]{2}{*}{ Std. Error } & \multirow[b]{2}{*}{ Sig. (2-tailed) } & \multicolumn{2}{|c|}{ 95\% Confidence Interval } \\
\hline & & & & & & Lower & Upper \\
\hline \multirow[t]{9}{*}{1} & (Constant) & 1.327 & -0.004 & 0.193 & 0.001 & 0.938 & 1.694 \\
\hline & Locations & 0.191 & 0.002 & 0.028 & 0.001 & 0.137 & 0.249 \\
\hline & Sector of work & -0.036 & 0.001 & 0.020 & 0.077 & -0.073 & 0.006 \\
\hline & Age (years) & -0.052 & -0.002 & 0.016 & 0.002 & -0.085 & -0.023 \\
\hline & Nationality & 0.434 & 0.000 & 0.114 & 0.001 & 0.225 & 0.670 \\
\hline & Sex & 0.164 & 0.000 & 0.084 & 0.054 & -0.007 & 0.326 \\
\hline & Practice area & -0.029 & 0.000 & 0.011 & 0.008 & -0.052 & -0.008 \\
\hline & Current Position & 0.070 & $-2.743 \mathrm{E}-05$ & 0.013 & 0.001 & 0.045 & 0.098 \\
\hline & Experiances & -0.042 & 0.001 & 0.042 & 0.327 & -0.122 & 0.040 \\
\hline
\end{tabular}


$(p=0.000)$. Regarding practice areas, the knowledge of reference management software was the lowest for emergency pharmacy (3.5128), with a statistically significant difference $(p=0.000)$. Based on years of experience, the lowest score (4.7126) was obtained for 11-15 years of experience, followed by $3-5$ years of experience (), with a statistically significant difference $(p=0.000)$. Based on the position held, the lowest score (4.0304) was obtained for community pharmacy, with a statistically significant difference $(p=0.000)$. The relationship between the knowledge of reference management software and factors affecting it was analyzed through a multiple regression model. The analysis revealed a weak relationship ( $\mathrm{R}=0.272$ with $p=0.000$ ) between the knowledge of reference management software and factors affecting it. Four out of eight factors showed non-significant differences $(p>0.05)$. However, nationality explained $20.3 \%$ of the positive relationship, practice area explained $18.9 \%$ of the negative relationship, current position held explained $18.7 \%$ of the positive relationship, and years of experience explained $16.1 \%$ of the negative relationship to the variation, with a statistically significant difference ( $p=0.0010 .001,0.003$, and 0.005 , respectively), which was confirmed by Bootstrap model. The relationship was verified by the nonexistence of multi-collinearity with the current position factor with VIF of $1.536,1.381,1.681$, and 1.397 , respectively, which is less than 3 or $5^{21-23}$ (Table 9).

\section{DISCUSSION}

Writing a research article or a manuscript is a critical part of the publication process. ${ }^{4-8}$ Academic writing is a required skill for all researchers. Each author had strengths and weakness points while writing the scientific studies. ${ }^{4-8}$ The declaration of the knowledge of publications demands setup plans, education, and training to improve the academic writing or publications skills. In this study, we explored the knowledge of pharmacists about scientific publications. We used a self-administered electronic survey questionnaire to obtain responses. The questionnaire was validated with a high-reliability test score. It was distributed to pharmacists locally, in different age groups, different working sites, qualifications, and years of experience. Most of the responders were from the central and eastern provinces, which was expected because of the author's location. Most of the responders had a medium level of work experience $(<6-10$ years) and were board-certified specialists, which is expected because the pharmacists might have had enough information about the requirements of a publication from their board, emphasizing research-writing skills for academic publications. The difference in demographic information of responders gave wide distribution of populations and different characteristics of various pharmacists. In addition, it gave the readers the background knowledge assessment from various pharmacists.

\begin{tabular}{|c|c|c|c|c|c|c|c|c|c|c|c|c|c|c|}
\hline \multicolumn{2}{|r|}{ Model } & $R$ & $\begin{array}{c}\mathrm{R} \\
\text { Square }\end{array}$ & $\mathrm{F}$ & Sig. & \multicolumn{2}{|c|}{$\begin{array}{l}\text { Unstandardized } \\
\text { Coefficients }\end{array}$} & $\begin{array}{c}\begin{array}{c}\text { Standardized } \\
\text { Coefficients }\end{array} \\
\text { Beta }\end{array}$ & $\mathbf{t}$ & Sig. & \multicolumn{2}{|c|}{$\begin{array}{l}95.0 \% \text { Confidence } \\
\text { Interval for B }\end{array}$} & \multicolumn{2}{|c|}{$\begin{array}{l}\text { Collinearity } \\
\text { Statistics }\end{array}$} \\
\hline \multirow[t]{7}{*}{1} & (Constant) & \multirow[t]{7}{*}{$.360^{\mathrm{b}}$} & \multirow[t]{7}{*}{.129} & \multirow[t]{7}{*}{7.431} & \multirow[t]{7}{*}{$.000^{\mathrm{b}}$} & 2.188 & 0.191 & & 11.463 & 0.000 & 1.813 & 2.563 & & \\
\hline & Locations & & & & & -0.078 & 0.025 & -0.159 & -3.197 & 0.001 & -0.127 & -0.030 & 0.882 & 1.134 \\
\hline & Age (years) & & & & & -0.013 & 0.017 & -0.039 & -0.774 & 0.439 & -0.045 & 0.020 & 0.844 & 1.186 \\
\hline & Nationality & & & & & 0.401 & 0.087 & 0.265 & 4.587 & 0.000 & 0.229 & 0.572 & 0.651 & 1.536 \\
\hline & Sex & & & & & 0.224 & 0.078 & 0.148 & 2.866 & 0.004 & 0.070 & 0.377 & 0.818 & 1.222 \\
\hline & Practice area & & & & & -0.009 & 0.009 & -0.057 & -1.043 & 0.298 & -0.027 & 0.008 & 0.724 & 1.381 \\
\hline & Current Position & & & & & 0.037 & 0.012 & 0.180 & 2.983 & 0.003 & 0.012 & 0.061 & 0.595 & 1.681 \\
\hline
\end{tabular}

a. Dependent Variable: Pharmacist's knowledge about journal indexing database, Predictors ${ }^{\text {b}}$ : (Constant), Location, Site of work, Age (years), Nationality, Pharmacist gender, Practice area, Current Position, and pharmacist experiences.

\begin{tabular}{|c|c|c|c|c|c|c|c|}
\hline \multicolumn{8}{|c|}{ Bootstrap for Coefficients } \\
\hline & \multirow{2}{*}{ Model } & \multirow{2}{*}{ B } & \multicolumn{5}{|c|}{ Bootstrap ${ }^{a}$} \\
\hline & & & Bias & Std. Error & Sig. (2-tailed) & \multicolumn{2}{|c|}{ 95\% Confidence Interval } \\
\hline \multirow[t]{6}{*}{1} & (Constant) & 2.188 & 0.011 & 0.234 & 0.001 & 1.727 & 2.648 \\
\hline & Locations & -0.078 & -0.001 & 0.022 & 0.001 & -0.125 & -0.037 \\
\hline & Nationality & 0.401 & 0.000 & 0.076 & 0.001 & 0.258 & 0.552 \\
\hline & Sex & 0.224 & -0.005 & 0.084 & 0.011 & 0.040 & 0.386 \\
\hline & Practice area & -0.009 & $-3.123 \mathrm{E}-05$ & 0.010 & 0.343 & -0.029 & 0.011 \\
\hline & Current Position & 0.037 & 0.001 & 0.012 & 0.001 & 0.014 & 0.060 \\
\hline
\end{tabular}


In this study, the average knowledge of writing an academic research article was acceptable, which agrees with the results of a previous study. ${ }^{19}$ This study emphasized sufficient knowledge about writing abstract and introduction with inadequate knowledge of other research sections such as discussions, limitations section, and reference. However, the finding was better than previous studies in Pakistan. ${ }^{22,30}$ That might be because pharmacists involve themselves in writing the abstract section, which is presented as a poster during their pharmacy education. ${ }^{9}$ However, some pharmacists did not have any publications or were not familiar with writing manuscripts, and the pharmacist used many electronic reference management software. The average score for knowledge of writing different types of articles based on study design was insufficient, with the highest knowledge obtained for observational study or case series study. This result was expected because most of the research projects required for graduation were to write a cohort, observational, or case study report. Moreover, the Pharm D students are required to present a case presentation. These skills were gained from clinical pharmacy rotation or advanced pharmacy practice experiences training. ${ }^{9}$

However, pharmacists were poor in writing meta-analysis, quality improvement, and animal studies, which might not be taught during their education and training at the college of pharmacy. Moreover, they continued their education in residency and masters' programs and did not learn by themselves in writing research articles. In this study, the average knowledge of pharmacists about indexing the database of publications was inadequate. The pharmacists were much more familiar with Google Scholar and PubMed, which is expected because most of them use it on a daily basis. At the same time, another database is not familiar with them because they seldom search about the literature through them. In this study, the average knowledge of using reference management software was satisfactory. Most pharmacists knew about reference management software (Mendeley and Qqica) because they were accessible and user-friendly. Moreover, the pharmacists did not use applications of references manager such as papers program.

\section{Factors affecting the knowledge of pharmacists about Scientific publications}

The knowledge of writing different sections of a research article was affected by various factors. Based on location, the northern region showed the lowest score related to insufficient education and training. The female gender showed greater knowledge than males, which might be because most female pharmacists were willing to publish their research than males. The pharmacy residency admission considered the publications as part of the admission evaluation score. $\mathrm{MOH}$ and the university as the worksites obtained low scores for writing publications,

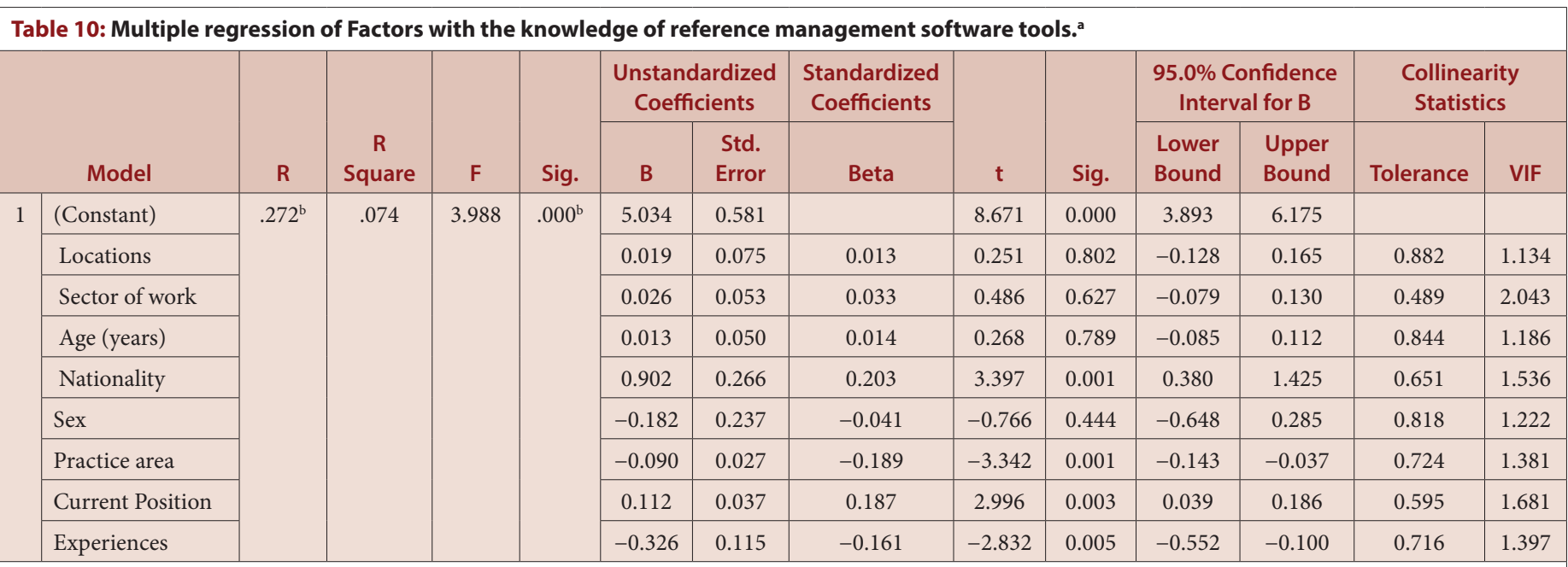

a. Dependent Variable: Pharmacist's knowledge of reference management software tools, Predictors ${ }^{\text {b}}$ : (Constant), Location, Site of work, Age (years), Nationality, Pharmacist gender, Practice area, Current Position, and pharmacist experiences.

\begin{tabular}{|c|c|c|c|c|c|c|c|}
\hline \multicolumn{8}{|c|}{ Bootstrap for Coefficients } \\
\hline \multirow{3}{*}{\multicolumn{2}{|c|}{ Model }} & \multirow{4}{*}{$\frac{\text { B }}{5.034}$} & \multirow{4}{*}{$\begin{array}{c}\text { Bias } \\
-0.037\end{array}$} & \multicolumn{3}{|c|}{ Bootstrap ${ }^{a}$} & \\
\hline & & & & \multirow[b]{2}{*}{ Std. Error } & \multirow[b]{2}{*}{ Sig. (2-tailed) } & \multicolumn{2}{|c|}{ 95\% Confidence Interval } \\
\hline & & & & & & Lower & Upper \\
\hline \multirow[t]{9}{*}{1} & (Constant) & & & 0.592 & 0.001 & 3.867 & 6.192 \\
\hline & Locations & 0.019 & -0.002 & 0.090 & 0.843 & -0.160 & 0.199 \\
\hline & Sector of work & 0.026 & -0.002 & 0.069 & 0.700 & -0.107 & 0.162 \\
\hline & Age (years) & 0.013 & 0.002 & 0.050 & 0.774 & -0.080 & 0.124 \\
\hline & Nationality & 0.902 & 0.008 & 0.332 & 0.007 & 0.246 & 1.502 \\
\hline & Sex & -0.182 & 0.008 & 0.269 & 0.507 & -0.697 & 0.351 \\
\hline & Practice area & -0.090 & 0.001 & 0.036 & 0.010 & -0.161 & -0.022 \\
\hline & Current Position & 0.112 & 0.002 & 0.038 & 0.006 & 0.040 & 0.197 \\
\hline & Experiances & -0.326 & -0.002 & 0.181 & 0.064 & -0.666 & 0.036 \\
\hline
\end{tabular}


which might be because the pharmacists were not interested, were busy working, or there was training provided during the college. Moreover, older age and more experience were negatively associated with the knowledge of writing publications. In the case of practice area, inpatient pharmacy was associated with less knowledge of writing publications. The higher position had the lowest knowledge because they were busy with pharmacy administration work or were not involved in a pharmacy strategic plan, or did not establish research and development services at the hospital pharmacy. This result is different from the regression analysis that's writing of section publications increase within 19\% with higher positions. It is expected to be included in the pharmacy continuous development program or research and development services at their healthcare institutions. Another depending factor was the location with a $16 \%$ increase with changing location emphasis the central region due to the availability of major healthcare organizations expert in scientific

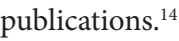

Various factors affected the knowledge of pharmacists about the study design used to publish the research. Location negatively affected the knowledge of pharmacists; the northern and southern regions showed the lowest scores. This result indicates inadequate knowledge of research designs and, subsequently, the writing of the research for publications. Females had more knowledge than males because they understood research design in a better way than males. Older age, more than 15 years of experience, and higher position held negatively affected the knowledge of research designs. This might be because of inadequate education and insufficient participation. Most of the pharmacists were busy with operation procedures of pharmaceutical care. They did not measure the impact of pharmacy services on the patients and economic outcomes. Other factors that affected the knowledge of research publications were worksite and $\mathrm{MOH}$; both showed low knowledge scores. That might be related to not being involved in the publication's procedures or inadequate education and training in writing research design. The Next practice site also negatively affected the knowledge of pharmacists about study designs. The inpatient pharmacy, pharmacy education, and medications safety were the lowest knowledge related to a busy workload to write study design reports. They did not measure the key performance indicators in various research designs or insufficient support of publications at healthcare organizations. According to the results of regression analysis, location, nationality, and current position held had a positive effect on the knowledge of study design. It is supposed to occur. Because of the higher positions had more implementation of scientific publications services emphasizing study design. The age of the responder showed a negative association. Furthermore, the practice site showed a negative association with the knowledge of study design.

Various factors affected the knowledge of pharmacists about journal indexing. The location of the pharmacist affected the knowledge of journal indexing. According to the results, western and southern regions showed low scores. Every journal has an ISSN number and DOI number, which is connected officially with the mega database Crossref, Google Scholar, and Microsoft Academic. Moreover, they might not be a part of journal evaluation. Most of the indexing is supported by the publishing companies, which might have lower knowledge of journal indexing with some worksite such as medical directorate in the regions and non$\mathrm{MOH}$ governmental hospitals. There are no international guidelines or recommendations from scientific societies around the world about the best or appropriate publications indexing database or journal indexing. In this study, nationality, young age, and less experience showed a positive association with knowledge of journal indexing because they needed to submit their publications to the residency program for admissions. Furthermore, females showed higher knowledge of journal indexing than males. Based on practice areas, such as satellite pharmacy primary care pharmacy, they had lower knowledge scores because of insufficient education and training. The higher position held showed a positive association with the journal indexing knowledge. The clinical pharmacist had inadequate knowledge of journal indexing because the clinical pharmacist did not publish any research due to their busy schedule. In the regression analysis, some factors were positive depending on nationality and gender. Other factors were negatively dependent such as location, age, and work experience. However, the current position showed a positive association on factors, which was supposed to occur due to higher academic qualifications and experiences to implement the scientific publication program. However, it was different from the practice, which was the insufficient implementation of writing articles. Various factors affected the knowledge of pharmacists about reference management software. Geographic location was one factor emphasizing the central and southern region had higher knowledge related to high publications at those areas or present of education and training about references manager. Next, non-Saudi nationals had more knowledge than Saudi nationals, which shows that pharmacists from foreign countries knew better about reference management software. Older age and more experience negatively affected the knowledge of reference management software. The emergency pharmacy practice area or community pharmacy positions showed the lowest knowledge of reference management software because of their heavy workload. The regression analysis showed positive dependent factors, such as nationality, with increasing knowledge of references section, which might be related to previous education and training at their countries. However, practice areas and years of experience negatively affected the knowledge of the reference section. However, one factor (positions) affected the results positively and increased the knowledge of reference manager software by higher jobs that are supposed to occur. However, it was different from our finding of real situations of responders.

\section{Limitations}

Although the results of this study were informative, it had some limitations. The demographic data of responders such as geographic locations, gender, age, working site, experiences, and career positions. Therefore, we recommend that future studies be conducted with equal demographic information among responders.

\section{CONCLUSION}

The knowledge of pharmacists about scientific publications is varied. The highest knowledge of writing section abstract, introduction, study design, and using of references manager. Various factors affected pharmacists' knowledge about scientific publications emphasizing that the older generation and more experience negatively affected the knowledge. Therefore, we highly recommend establishing research and development programs at pharmacy centers to improve knowledge of scientific publications in pharmacy practice in Saudi Arabia.

\section{ACKNOWLEDGEMENT}

None.

\section{CONFLICT OF INTEREST}

The authors declare that there is no conflict of interest.

\section{Funding}

None

\section{Consent for Publications}

Informed consent was obtained from all the participants 


\section{Ethical Approval}

This research is exempted from research and ethical committee or an institutional review board (IRB) approval.

https://www.hhs.gov/ohrp/regulations-and-policy/decisioncharts-2018/index.html

\section{ABBREVIATIONS}

MOH: Ministry of Health; KSA: Kingdom of Saudi Arabia; SPSS: Statistical package of social sciences; JASP: Jeffery's Amazing Statistics Program; STROBE: Strengthening the reporting of observational studies in epidemiology; ADR: Adverse Drug Reaction.

\section{ORCID ID}

Yousef Ahmed Alomi (iD https://orcid.org/0000-0003-1381-628X

\section{REFERENCES}

1. Cuschieri S, Grech V, Savona-Ventura C. WASP (Write a Scientific Paper): Structuring a scientific paper. Early Hum Dev. 2019;128:114-7. doi: 10.1016/j. earlhumdev.2018.09.011, PMID 30236948

2. Liumbruno GM, Velati C, Pasqualetti $P$, Franchini M. How to write a scientific manuscript for publication. Blood Transfus. 2013;11(2):217-26. doi: 10.2450/2012.0247-12, PMID 23356975.

3. International Committee of Medical [journal]. Recommendations for the conduct, reporting, editing, and publication of scholarly work in medical journals [internet]. International Committee of Medical [journal. p. 2019]. Available from: http://www.icmje.org/icmje-recommendations.pdf.

4. Schulz KF, Altman DG, Moher D, CONSORT. Statement: Updated guidelines for reporting parallel group randomized trials. BMJ. 2010;340(7748):698-702.

5. Von Elm E, Altman DG, Egger M, Pocock SJ, Gøtzsche PC, Vandenbroucke JP The strengthening the reporting of observational studies in epidemiology (STROBE) statement: Guidelines for reporting observational studies. PLOS Med. 2007;4(10):1623-7. doi: 10.1371/journal.pmed.0040296.

6. Von Elm E, Altman DG, Egger M, Pocock SJ, Gøtzsche PC, Vandenbroucke JP. The Strengthening the Reporting of Observational Studies in Epidemiology (STROBE) statement: Guidelines for reporting observational studies [internet]. Vol. 370; 2007. Available from: http://www.thelancet.com. Available from: http://www.plosmedicine.org [cited 23/11/2021].

7. Langan SM, Schmidt SA, Wing K, Ehrenstein V, Nicholls SG, Filion KB, et al. The reporting of studies conducted using observational routinely collected health data statement for pharmacoepidemiology (RECORD-PE). BMJ. 2018;363:k3532. doi: 10.1136/bmj.k3532, PMID 30429167.

8. Simera I, Moher D, Hoey J, Schulz KF, Altman DG. A catalogue of reporting guidelines for health research. Eur J Clin Investig. 2010;40(1):35-53. doi: 10.1111/j.1365-2362.2009.02234.x, PMID 20055895

9. Asiri YA. Emerging frontiers of pharmacy education in Saudi Arabia: The metamorphosis in the last fifty years. Saudi Pharm J. 2011;19(1):1-8. doi: 10.1016/j.jsps.2010.10.006, PMID 23960737.

10. Kheir N, Zaidan M, Younes H, El Haij M, Wilbur K, Jewesson PJ. Pharmacy education and practice in 13 middle eastern countries. Am J Pharm Educ. 2008;72(6):133. doi: 10.5688/aj7206133, PMID 19325953.

11. Al-Haidari KM, Al-Jazairi AS. Establishment of a national pharmacy practice residency program in Saudi Arabia. Am J Health Syst Pharm. 2010;67(17):1467-70. doi: 10.2146/ajhp090536, PMID 20720247

12. Al-Qadheeb NS, Alissa DA, Al-Jedai A, Ajlan A, Al-Jazairi AS. The first international residency program accredited by the American Society of Health System Pharmacists. Am J Pharm Educ. 2012;76(10):190. doi: 10.5688/ajpe7610190, PMID 23275655.
13. The general directorate for research and studies (GDRS-MOH). Anuual Reserach Repot. 2020;19-20.

14. Alhaider I, Mueen Ahmed KK, Gupta BM. Pharmaceutical research in the Kingdom of Saudi Arabia: A scientometric analysis during 2001-2010 [internet] Saudi Pharm J. 2015;23(3):215-22. doi: 10.1016/j.jsps.2013.07.008, PMID 26106268.

15. Streetman DS, Mccreadie SR, Mcgregory M, Ellis JJ. Evaluation of clinical research knowledge and interest among pharmacy residents: survey design and validation. Am J Heal Pharm-Vol. 2006:63(23):2372-7. doi: 10.2146/ ajhp060099, PMID 17106011.

16. Awaisu A, Bakdach D, Elajez RH, Zaidan M. Hospital pharmacists' self-evaluation of their competence and confidence in conducting pharmacy practice research. Saudi Pharm J. 2015;23(3):257-65. doi: 10.1016/j.jsps.2014.10.002, PMID 26106274.

17. Alomi $Y$, Alabdullatif $A$, Alharbi $A$, Altebainawi A. Basic pharmacy research knowledge in the Kingdom of Saudi Arabia. IJMDC. 2020 (September):1216-25. doi: 10.24911/IJMDC.51-1592548068.

18. Alomi YA, Altebainawi AF, Alharbi AA, Alabdullatif AA. Biostatistical analysis knowledge of pharmacy research in the Kingdom of Saudi Arabia. Int J Adv Appl Sci. 2017;14(3):18.

19. Wager E, Woolley K, Adshead V, Cairns A, Fullam J, Gonzalez J, et al. Awareness and enforcement of guidelines for publishing industry-sponsored medical research among publication professionals: The Global Publication Survey. BMJ Open. 2014;4(4):e004780. doi: 10.1136/bmjopen-2013-004780, PMID 24747794

20. Schroter S, Roberts J, Loder E, Penzien DB, Mahadeo S, Houle TT. Biomedical authors' awareness of publication ethics: an international survey. BMJ Open. 2018;8(11):e021282. doi: 10.1136/bmjopen-2017-021282, PMID 30478105.

21. Punyani SR, Deshpande A. Authors' awareness of concepts in the authorship of scientific publications: Viewpoints of the dental faculty in India. J Oral Biol Craniofac Res. 2018;8(3):151-3. doi: 10.1016/j.jobcr.2016.05.001, PMID 30191098

22. Hussain M, Rehman R, Baig M. Manuscript writing and publication workshop: An invoking pilot study on enhancing cognitive research capabilities in Health Sciences Institutes of Pakistan. Cureus. 2020;12(6):e8802. doi: 10.7759/ cureus.8802, PMID 32724748.

23. Charan J, Biswas T. How to calculate sample size for different study designs in medical research? Indian J Psychol Med. 2013;35(2):121-6. doi: 10.4103/02537176.116232, PMID 24049221.

24. Pourhoseingholi MA, Vahedi M, Rahimzadeh M. Sample size calculation in medical studies. Gastroenterol Hepatol Bed Bench. 2013;6(1):14-7. PMID 24834239.

25. Ezhumalai DG. How Big A Sample Do I Require?. Annals of SBV. 2017;6(1):39-41. doi: 10.5005/jp-journals-10085-6113.

26. Johnson TP, Wislar JS. Response rates and nonresponse errors in surveys [internet]. JAMA. 2012:307(17):1805-6. doi: 10.1001/jama.2012.3532, PMID 22550194.

27. Liao $D$, Valliant R. Variance inflation factors in the analysis of complex survey data. Surv Methodol. 2012;38(1):53-62.

28. Akinwande MO, Dikko HG, Samson A. Variance inflation factor: As a condition for the inclusion of suppressor variable(s) in regression analysis. Open J Stat. 2015;05(7):754-67. doi: 10.4236/ojs.2015.57075.

29. Thompson CG, Kim RS, Aloe AM, Becker BJ. Extracting the Variance Inflation Factor and Other Multicollinearity Diagnostics from Typical Regression Results. Basic Appl Soc Psych. 2017;39(2):81-90. doi: 10.1080/01973533.2016.1277529.

30. Bhagavathula AS, Bandari DK, Jamshed SO, Chattu VK. Pharmacy students perception and inclination toward scholarly research publications: A multinational study. J Educ Health Promot. 2018;7:131. doi: 10.4103/jehp.jehp_77_18, PMID 30505859 . 